\title{
Control Techniques for Active Power
}

\section{Filters}

\section{Abstract}

There have been many variants of the active power filter proposed and these variations cover both the circuit topology and the control system employed. Some of the control variants reflect different control objectives but there are still many variants within similar objectives. This paper describes and contrasts the available control techniques in a structured way to identify their performance strengths. Objectives are classified by the supply current components to be corrected and by the response required to distorted grid voltage. The various signal transformations are described in terms of their impact on the distortion identification problem. Time-domain, frequency-domain, instantaneous power and impedance synthesis methods are examined. Additional control functions such as DC-bus voltage and current reference following are also discussed. It is found that a key difference between control methods is way in which current distortion is treated in the presence of distorted grid voltage.

\section{Introduction}

The idea of active filtering of distortion found in power distribution lines appears in the literature from the 1970s [1,2,3,4]. The terms active filter and active power filter (APF) are both in common use. Here active power filter is preferred to distinguish filter that must process instantaneous power from active filters for signal processing. Since the early schemes, many Active Power Filter (APF) variants have been proposed and the literature has been reviewed from several stand points. An early review was [5]. It categorises active power line conditioners according to whether time or frequency domain signal processing is used and whether current or voltage type converters are used. Akagi reviewed the emerging APF technologies [6] in terms of their objectives, configuration and controllers and discussed the unified power quality conditioner (a combination of a shunt and a series APF). 
In [7], Peng reviewed the literature regarding shunt versus series forms of APF and articulated clearly the need to match the form of filter to the form of distortion. Thus, shunt APFs are effective against current-stiff (inductive) non-linear loads that inject distortion current whereas series APFs are effective against voltage-stiff (capacitive) non-linear loads that inject distortion voltage. While it is possible to use the other combinations, the ratings required of a shunt APF when used to compensate a current-stiff non-linear load can become large if the line impedance is small [7, 8]. In [9] Peng develops the theme and expands the discussion to the several forms of hybrid active/passive power filters (Hybrid APFs) appearing in the literature. The paper uses the idea of duality to fully populate the matrix of possible combinations of shunt and series impedances and controlled sources. The features and operating characteristics of each combination are examined. Another categorisation of topologies for hybrid filters was presented in [10].

Singh et al. [11] categorise a large number of reported active filters under headings of Converter, Topology, Supply Lines, Signal Conditioning and Compensation Derivation. El-Habrouk et al. [12] categorise published work under headings of Power Rating; Circuit Configuration; Compensation Quantity, Control Method and Reference Identification. The assessment under each heading is necessarily brief but the reviews are comprehensive.

There are several papers that compare certain control schemes for the quality of the results they produce such as $[13,14]$. Execution speed and time response of three harmonic identifiers were reviewed in [15].

This paper draws together the various control schemes reported and identifies their characteristics. The literature on APFs contains reports of many different circuit topologies but these are not a focus of this review. To simplify the discussion here, attention is focused on the case of a shunt active filter injecting compensating current into a line. A control method for a shunt filter can normally be applied to a series compensation case using the ideas of duality discussed in [9]. Only where a control method is tailored to a different circuit topology, is that topology example used. APF control includes subtasks such reference generation, current control and DC-bus voltage control. Each of these topics is covered. The focus is on three-phase methods as the more general situation. Where a method can be applied with modification to a single-phase APF this is discussed. 


\section{APF Structure}

Figure 1 shows the shunt connected APF used as the principal example and illustrates the five basic elements of an APF:

1. Distortion Identifier - a signal processing function that takes the distorted waveform (the line current or voltage), $d(t)$, and forms a reference waveform, $r(t)$, which will reduce the distortion.

2. Inverter - a power converter (and coupling inductance/transformer) able to reproduce the reference waveform at suitable amplitude, $I_{A P F}$ (or $\left.V_{A P F}\right)$.

3. Inverter Controller - a pulse-width modulator and, in the case of a voltage source inverter used to inject current, a local current control loop that ensures that $I_{A P F}$ tracks $r(t)$

4. Synchroniser - a signal processing block based on phase-locked loop techniques that ensures that the cancellation waveforms are correctly synchronised to the mains voltage. Certain methods do not require explicit synchronisation.

5. DC-Bus - an energy store that supplies the fluctuating instantaneous power demand of the inverter. Errors and losses that cause the energy store to engage in long term real power flows must be compensated for by additional action of the inverter controller.

An example series APF is shown in figure 2. It uses the same basic elements but the inverter is configured to inject series voltage. According to the classification in [6], Figure 1 would be described as current injecting and load current sensing. Figure 2 would be described as voltage injecting and load current sensing. Voltage detecting configurations are also possible and can be used with voltage or current injection.

The system shown in Figure 1 has been described as open-loop in the discussion in [16]. It is open-loop in that $i_{d}$ (not $i_{s}$ ) is measured. The distortion generated by the load is then identified and a correction term fed-forward to correct the distortion in the supply. There may be errors in the calculation process or inaccuracy in the current injection that result in imperfect correction. There may also be some coupling such that the injected current perturbs the source or the load such that the distortion changes and the correction is unstable. The calculation of the feed-forward correction is subject to processing delay and so the transient response is a concern. (Although Figure 1 contains a control loop, it is a local loop to operate the voltage-source inverter as a current source.)

In contrast, the closed-loop approach measures $i_{s}$, identifies any remaining distortion and updates the injection reference. In some cases this update will be a low sample rate process with perhaps one update per mains period. 
This requires that the update coefficient (gain) is low so that a stable approach is made to the zero-distortion target [16]. Slow convergence of this loop provides a degree of smoothing in the compensation current of a fluctuating distortion load. Continuous versions of closed loop control have also been reported but with a relatively slow adjustment of the target [17].

\section{Choice of Cancellation Objective}

In principle, an APF is capable of correcting a wide variety of power quality problems such as:

- Harmonic Distortion (of any phase sequence)

- Fundamental-Frequency Reactive Power (non-unity displacement factor)

- Negative Sequence Fundamental Components (unbalance components)

- Zero-Sequence Fundamental Components (neutral line current)

- Flicker (low frequency modulation of power flow)

Correction of harmonic distortion is taken as a core function present in all APFs. Displacement factor correction with fundamental frequency reactive power is often also included [18]. Correction of the fundamental frequency negative-sequence component can be provided and will balance unbalanced load currents in a three-wire system [19]. With a four-wire system, zero-sequence harmonics and zero-sequence fundamental can flow as a result of single-phase distorting loads and a four-wire APF can be used to correct these terms. Flicker correction is normally a function of a Dynamic Voltage Restorer (DVR) but can fall within the remit of an APF [20]. Functions such as combating voltage sag and swell are considered to be DVR functions. The use of an APF in response to various power quality problems is discussed in [21].

It is tempting to consider that all of the various power quality issues can be dealt with by simply adding control functions to the basic APF power converter circuit. However, each corrective action contributes to the voltampere rating of the power converter and hence to the cost of the equipment. Compensation of unbalance and flicker also has implications for the DC-side energy store since the energy flows represented by peaks in the instantaneous power can be large. With this acknowledged, it is important to note that a control scheme designed to correct harmonic distortion might provide unintentional action to correct flicker and thereby cause the APF to exceed its rating when faced with large flicker components. Control schemes need to be assessed for their effectiveness in correcting the problems they were explicitly designed for and assessed for any unintended 
additional action. The largest energy exchanges with the DC-side are likely to arise from fundamental frequency unbalance and low frequency power variation (flicker).

Any discussion of APF control rests on the definition of the distortion that is to be corrected. Viewed from the standpoint of an ideal power system with balanced sinusoidal voltages, the ideal load current is also a balanced sinusoid set. A non-sinusoidal current or an unbalanced current can be decomposed and the distortion terms identified. This identification of distortion can also be approached from an analysis of the instantaneous power. For the case of a sinusoidal voltage and distorted current, identification of the active power is equivalent to identification of the in-phase fundamental component of current. For the general case of an unbalanced, nonsinusoidal voltage and an unbalanced non-sinusoidal current there is still debate over the most appropriate form of decomposition. There are two long standing approaches. Fryze [22] defined active current, $i_{A}(t)$ as being responsible for the real power flow and having the same form as the voltage, (1). The active current is the minimum RMS current required to transmit the active power. The remaining current was defined as non-active current, $i_{N}(t)$ (2) and is found to be orthogonal to the active current (3).

$i_{A}(t)=\frac{P}{\|v\|^{2}} v(t)$

where $\|\bullet\|$ is the RMS value and $\mathrm{P}$ is the real (average) power.

$i_{N}(t)=i(t)-i_{A}(t)$

$\|i\|^{2}=\left\|i_{A}\right\|^{2}+\left\|i_{N}\right\|^{2}$

The Fryze non-active power, $Q_{F}$ and the apparent power, $\mathrm{S}$ are defined by multiplying the current equation by the RMS voltage (4)

$S^{2}=P^{2}+Q_{F}^{2}$

Budeanu used a frequency domain decomposition of voltage and current to define the active (5) and reactive powers (6).

$$
\begin{gathered}
P=\sum_{n=1}^{N} V_{n} I_{n} \cos \left(\phi_{n}\right) \\
Q_{B}=\sum_{n=1}^{N} V_{n} I_{n} \sin \left(\phi_{n}\right)
\end{gathered}
$$

A third power term, $D$ needs to be introduced to complete the apparent power equation (7). 
$S^{2}=P^{2}+Q_{B}{ }^{2}+D^{2}$

Enslin and Van Wyk surveyed possible approaches to decomposing power into terms that can be separate out for compensation (or not) [23, 24]. There are several other views of this topic [25] and much debate [26]. Peng [27] has shown the relationship between the definition of active power and the definitions of instantaneous active and reactive power that become popular in APF applications (to be reviewed in Section 6)

The shunt current-correcting APF will often be placed in the low voltage distribution network where there is significant degree of existing voltage distortion caused by distant non-linear loads and their common-impedance coupling to the APF site. A significant question to address is what is the most desirable response of the combined APF+load to the distortion components of the supply voltage. As will be shown in section 5, many of the reported methods set an objective of achieving sinusoidal current flow. This can be interpreted as presenting a very high impedance to harmonic voltages (and a moderate impedance to the fundamental term). In contrast, the instantaneous power method (to be described in Section 6) draws significant harmonic current in response to harmonic voltages. The response is complex and as will be shown in section 6 , the process is non-linear and results in current harmonics at different frequencies to the voltage excitation. The non-sinusoidal current has been noted and several attempts have been made to force sinusoidal current. However, [28] and [29] argue (from differing starting points) that the APF should respond to voltage distortion but should do so with a resistive characteristic. Harmonic currents will be drawn in phase with the harmonic voltage excitation and the power extracted by the $\mathrm{APF}+$ load will provide damping of the excitation. If the same resistance is presented at all frequencies then the current waveform will have the same shape as the voltage waveform. This matches the definition of active current discussed by Fryze and used in [23]. Three categories of compensation can be defined.

1. Waveform Compensation. Commonly the objective is to achieve a supply current with fundamental active current only. In impedance terms, the APF+load is resistive at fundamental frequency and open circuit at harmonic frequencies.

2. Instantaneous Power Compensation. Commonly the objective is to achieve a constant instantaneous power drawn from the supply. The APF+load present a complex, non-linear response to distorted excitation and can not be described in terms of an impedance. 
3. Impedance Synthesis. Commonly the objective is to present a resistive characteristic. Power is absorbed at all frequencies present in the excitation and APF+load can be made to closely approximate a passive system.

\section{Waveform Compensation}

Waveform correction can be approached in many ways. There are many signal processing techniques that can decompose the current waveform into various components and separate those that should remain from those that should be cancelled (the cancellation reference). This is essentially a filtering task and the non-ideal properties of real filters must be recognised. In addition to the analytical approaches, there are pattern learning techniques such as neural networks.

\subsection{Filter-based methods}

Distortion identification is a signal filtering task and can be conducted with time domain filters or by Fourier based frequency decomposition. As with all filtering tasks, the filters need to be considered in terms of the following:

- Attenuation: it is important that the identified components have their magnitude preserved, that the other components are heavily attenuated and that the transition band between the pass and stop bands is narrow

- Phase-distortion: because the cancellation relies on injecting cancellation signals in phase-opposition, it is important to preserve the phase of the identified components

- Time-response: the (distorted) load current will be subject to change and the filter should respond rapidly without large overshoot.

These three performance considerations are inextricably linked for time-domain filters: flatness of magnitude response has to be traded off against phase-response (at least for causal filters) and a well-damped transient response is in conflict with a narrow transition region.

Applying a filter to determine directly the distortion means that the cancellation process is subject to the transient response of the filter. The alternative is indirect identification, i.e., use the filter to determine the fundamental signal $f(t)$ that should remain and subtract that from the instantaneous distorted signal $d(t)$ to form 
the reference $r(t)$ (illustrated in Figure 3). There are two stages of inversion in achieving the desired component: first it is subtracted from the measured current to form the reference and then the reference is subtracted from the actual line current. The difference between the direct and indirect methods is apparent during a transient. Real-time filters are causal and during a transient, there is a time lag between the output of the filter and the component to be identified. Thus, the direct method will have an out-of-date distortion term and the distortion cancellation will be in error. The indirect method will have an out-of-date fundamental term and, while the distortion terms will be cancelled, real power will also be exchanged through the inverter. Real power exchange disturbs the DC-bus voltage and requires that the inverter be rated to cope with a real power component which might be large.

When considering unintended action of a distortion identifier it must be borne in mind that for a direct identifier, only those components specifically separated by the signal filter will be compensated by the APF whereas for an indirect identifier, any component not specifically identified by the signal filter for retention will be compensated by the APF.

Most APF implementations opt for indirect distortion identification since it yields the best distortion cancellation during transients. The inadvertent exchange of real power (caused by incomplete separation of the real power component in an indirect identifier) can be corrected as explained in section 8. Direct identification is used where different groups of harmonics are to be treated differently or where only a specific range of harmonics are to be compensated [19]. This might be desirable where this allows the rating of the filter to be kept within a limit or where interaction with a system resonance is a danger if cancellation is attempted in a certain frequency band.

The well known transformations of three-phase systems are widely used in APF controllers to facilitate separation of a current or voltage term for cancellation. The transform to orthogonal components in a stationary reference frame (the $\alpha \beta 0$ reference frame) and the transform to a rotating reference frame (the $d q 0$ reference frame) are both useful. The choice of reference frame in which to operate has an important impact on the filter design; in particular:

- The width of the transition band between pass-band and stop-band is different in different domains 
- The decomposition of the fundamental into various components depends on the domain used and gives choice over what type distortion is cancelled.

Both of the transforms separate out the zero-sequence component into a separate term (or axis). The $\alpha \beta 0$-transform does not separate sequence-sets (although the sequence can be determined from whether the $\alpha$ or $\beta$ component leads). The rotation transformation shifts fundamental frequencies to DC and separates out the active and reactive components. Components of the same harmonic order but of opposite rotation (phase sequence), i.e., $+\mathrm{n}$ and $-\mathrm{n}$, are separated from each other because positive sets are shifted to order $\mathrm{n}-1$ and negative sets to order $-\mathrm{n}-1$.

An early example of using the synchronous frame is [30,31]. In [32] it is argued that, especially for high sample rate systems, the overhead in performing $\alpha \beta 0$ and rotation transformations is too much of a burden. A method is demonstrated of transforming a filter designed in a rotating frame to an exact equivalence in the stationary frame. Importantly, the method preservers the ability of a synchronous frame filter to distinguish between negative and positive sequence sets through the retention of coupling between the axes.

Figure 4 shows the form of the spectrum expected from a distorting load as it would in each of the domains being discussed. The load is assumed to be three-wire, non-linear and unbalanced. Figure 4(a) is the spectrum of a phase-current and shows the expected harmonic distortion of a non-linear 3-wire, 3-phase load, i.e., harmonics of order $6 k \pm 1$ (where $k$ is any positive integer).

Figure 4(b) shows the spectrum of the currents transformed by the $\alpha \beta$-transform. This has no effect on the frequencies present but, with all three-phases represented, the phase-sequence can be observed in the phase relationship between the $\alpha$ and $\beta$ components. In this figure, negative sequence terms are shown as having a negative frequency (i.e., to be backward rotating). Thus, the unbalance of the fundamental is shown by the presence of a harmonic of order -1 . Balanced non-linear loads produce characteristic harmonics of order $-5,+7$, $-11,+13, \ldots 6 k+1$ (where $k$ is any integer). Unbalanced non-linear loads produce further non-characteristic harmonic distortion of order $+5,-7,+11,-13, \ldots 6 k-1$. 
Figure 4(c) shows the spectrum of the signal after the rotation transform has been applied. All components have been shifted down one harmonic order. The positive sequence fundamental becomes a DC term whereas the negative sequence fundamental becomes a double frequency term (order -2). The characteristic distortion is of order $6 k$ and the non-characteristic distortion of order $6 k-2$

There is no difference between filtering in the phase- or $\alpha \beta 0$-domains but the $d q 0$-domain does offer an alternative. In the phase-domain the fundamental can be separated (for indirect identification of the harmonics) with a low-pass filter that cuts-off between orders 1 and 5 ( 1 and 3 if there is zero-sequence / 4-wire harmonic distortion). In the $d q$-domain the filter cut-off should be between 0 and 6 for balanced conditions. For unbalanced conditions a cut-off between 0 and 2 will allow negative sequence fundamental (unbalanced fundamental) to be corrected and a cut-off between 2 and 4 will not cancel unbalanced fundamental but will still cancel harmonic distortion. Zero-sequence distortion conveniently appears in a separate term and can be cancelled or not as required. Thus, the $d q$-domain offers frequency separation of unbalance components and the placement of key components at DC where they are relatively easy to filter.

Filters that are required to pass the DC term alone (in the $d q 0$-domain) can be designed as low-pass filters with very low cut-off frequencies and more than a decade of transition region. It is important to realise that using a very low cut-off frequency will mean that flicker components in the current will not be retained in the desired waveform and will be cancelled by the APF. The cancellation of flicker components might be desirable but there will be substantial power exchange between the load and the DC-bus of the APF with implications for the ratings of both the inverter and the DC energy store.

Filters that are required to pass fundamental and reject higher terms (an indirect identifier in the phase- or $\alpha \beta 0$ domain) can in principle be a low-pass filter since no DC term is expected. However, it is not possible to obtain satisfactory pass- and stop-band performance from a practical low-pass filter with a transition band as narrow as a few harmonic orders. However, a notch filter with a very narrow notch can be realised. There can be difficulties, too, with notch filters [33]. If the system frequency is subject to wide variation the width of the notch must be compromised. Selectable filters will be needed to cover both $50 \mathrm{~Hz}$ and $60 \mathrm{~Hz}$ operation. 
Because the pass-band and stop-band are separated by only a few harmonic orders it is inevitable that there will be incomplete separation between distortion and the allowed components. In a direct identifier, pass-band errors in magnitude or phase will lead to incomplete cancellation of distortion whereas incomplete rejection in the stop-band will lead to the APF exchanging some fundamental real and reactive power with the AC system. In an indirect identifier the situation is reversed: errors of phase or magnitude in the pass-band of the filter lead to the exchange of fundamental frequency real and reactive power and incomplete rejection in the stop-band leads to incomplete cancellation of harmonics. The use of high-order filters to provide a narrower transition band has the disadvantage of greater phase error and a more complex transient response.

\subsection{Frequency-domain filtering}

Filtering in the frequency domain is somewhat different from filtering in the time-domain and involves a Discrete or Fast Fourier Transform (DFT or FFT) on a block of data that contains at least one cycle of the lowest frequency of interest and that has been sampled at over twice the highest frequency present. The advantage of filtering in the frequency domain is that completely abrupt cut-offs (with no transition band) can be obtained. Pass-band ripple and phase distortion can be avoided provide care is taken over the transformation into and out of the frequency domain in terms of synchronisation, buffer length and window function. The accompanying disadvantage is that frequency domain filters are not real time filters. Time must elapse for sufficient samples to be gathered and, when that is complete, processing time is required to perform the transform.

As discussed in [16], the signals to be processed must be steady and periodic before frequency-domain processing is appropriate. The FFT implicitly assumes periodicity of the sampled waveform. If the FFT window is properly synchronised to the fundamental signal then the phase and magnitudes of the components can be accurately determined. If the window does not cover an integer number of fundamental cycles then spectral leakage will occur and accuracy is degraded and windowing functions will be required to partially overcome this problem. The selectivity of Fourier methods with respect to time domain filters is one of the aspects of the discussion in [19].

Figure 5 shows direct and indirect distortion identification using frequency domain filtering. In the direct method the fundamental component is set to zero to form a cancellation reference for harmonic distortion. If only the real (in-phase) component is set to zero then the APF will also cancel the reactive component. In the 
indirect case the harmonic terms are set to zero so that the fundamental is subtracted from the instantaneous signal to yield the reference. If the imaginary (quadrature) fundamental component is set to zero then it will not be subtracted from the instantaneous signal and will be cancelled by the APF. In [19], the harmonic identifier used a direct method in which individual components were separately identified. For certain harmonics, the compensation current and the load current were compared and integrator action applied to any error. Where it was judged that this closed loop control might interact with a resonance in the electrical system, it was omitted and open loop control was used.

During a transient the periodicity of the distortion is lost and the cancellation is not accurate. In addition, there is a time delay in the filtering that affects the distortion identification. In the simple case, one complete cycle of data is required which is stored in a buffer and then processed during the following cycle and de-buffered for use as a cancellation reference in the cycle after that. Thus, the cancellation reference is two cycles out-of-date. Shorter update rates are possible such as performing an FFT every half-cycle using one half-cycle of fresh data plus data from the previous half-cycle. The delay is then one complete cycle. Faster updates are possible limited by the computational power available to process data in real time. De-buffering can present problems with the continuity of the resulting waveform. Full or half cycle buffers aligned to the zero-crossings of the fundamental can be de-buffered to yield a continuous signal. Buffer edges at other points (smaller buffers or buffers of threephase data aligned to one particular phase) can cause a step change in the cancellation reference during transient conditions. A smoothing function can be applied to the buffer edges but response times are affected.

\subsection{Heterodyne Methods}

The heterodyne method of waveform compensation involves multiplying a distorted signal by a sinusoid. If the sinusoid is at fundamental frequency then the fundamental frequency component that is in phase with heterodyning signal transforms to a DC term and a double frequency term. The DC term can be separated with a low-pass filter. If the heterodyning sinusoid is in phase with the voltage then this method will identify the fundamental active current. The fundamental reactive current could be identified by using a quadrature sinusoid. This is a method of signal processing that has become popular in single-phase systems where $\alpha \beta 0$ and $d q 0$ transforms are not applicable. A discussion of the method is presented in [34]. 
Heterodyning can be applied to three-phase systems by using a phase-locked loop to form a sinusoid locked to the fundamental of the supply voltage [35]. The choice of filter is a compromise: the DC term must be passed and the double frequency term rejected. A sharp, high-order filter will give a long step response whereas a loworder filter will give poor separation of the terms and imperfect current cancellation. In [35] the identification was done in closed-loop with integral action. This overcame parameter errors in the system. The resulting system had a response time of 2-3 mains cycles.

The $d q 0$ transformation, as already discussed, is a form of heterodyning with the fundamental frequency that exploits the properties of a balanced three-phase set. The advantage of the $d q 0$ transform over applying heterodyning to each phase is that the double fundamental frequency term is eliminated if the variables are balanced and the filter need not be designed to reject this term. The filter can be designed to remove only the higher order terms. The $d q 0$ transform has been applied to individual phases as a form of heterodyning [36] by using time-delay/phase-shifting to form pseudo three-phase sets.

A DFT can be considered as a succession of heterodyne operations for each harmonic followed by averaging to filter out all unwanted components. In the classification here, the heterodyne category is reserved for methods that select out a small number of distortion components using heterodyne techniques. In [15] a method described as a recursive DFT was studied. It is equivalent to a heterodyne followed by moving average filtering. It was shown to be computationally efficient compared to other methods provided that the number of terms required was small. The method in [37] is described in terms of an adaptive filter that eliminates the fundamental from the load current signal to yield the cancellation reference. In fact, the adaptive element is heterodyning function followed by integration to detect the fundamental term.

Direct identification methods using multiple synchronous reference frames, each synchronous with a particular harmonic of interest, have been reported [38, 39]

\subsection{Pattern Learning and Identification}

Because of the time-delay in frequency-domain identification and the compromises in filter design in timedomain based methods, several attempts have been made to use pattern learning to perform the separation of a 
current into a portion to cancel and a portion that should remain. The direct and indirect principles can both be used.

An early attempt to use a neural network as an harmonic identifier is reported in [40]. A 2-layer neural network was used to estimate the Fourier coefficients of a distorted waveform. A digital implementation is described but it is doubtful that it is more computationally efficient than an FFT.

An early application of a neural network to an APF was arranged to learn the characteristics of the local grid system such that a suitable correction current could be set to achieve a target level of voltage distortion in the grid [41]. It was anticipated that several distorting loads exist and that measurement of load current was not feasible. The harmonic components of the supply voltage are found by conventional means and the network conductance used to assess the appropriate current. This current is the training reference for the neural network. As the neural network adapts, the injected current is adjusted until the voltage error is driven to zero. A separate network is required for each harmonic frequency. This technique was assessed within [42]. Successful operation was confirmed but it was noted that the response to a change in load took more than 10 mains cycles to converge. From this, it was proposed in [42] to combine a traditional harmonic identifier acting on the measured load current and to supplement this with the neural network acting on the voltage error.

Also examined in [42] is a neural network that accepts time-domain samples of load current (as a vector of timedelayed samples covering a cycle) and is trained to produce the cancellation reference as time-domain samples. Simulation results show that this can achieve acceptable results with some useful generalization around the training data. However, it is noted that the network requires a relatively large number of hidden-layer neurons and has correspondingly large training time and recall time. A different arrangement of the time-domain method is presented in $[43,44]$. Here the network again uses a vector of time-delayed load current samples as its input but it has been trained to identify the active component of current that should remain in the supply after cancellation.

The ambition of using a neural network to provide fast frequency decomposition for an APF is explored in [45]. A network was trained to identify the $3^{\text {rd }}$ and $5^{\text {th }}$ harmonic cosine coefficients for a range of load current amplitudes. This is a direct algorithm. The algorithm can be applied to each half-cycle of load current data and 
simulation testing shows an accuracy of better than $1 \%$ using 100 samples and 10 middle layer neurons. The computational effort in recall is not identified but the network is relatively small because only two terms are identified. The estimation of Fourier series terms is also undertaken by an ANN in [46]. The fundamental active power is then calculated and a fundamental current reference formed. A further ANN is used to implement the selection of inverter states to force current reference following. Results are given for an example 4-wire system that show the system makes response to a change in load during the following cycle and achieves its full response after 2-3 cycles.

A neural network has been used in an indirect algorithm to identify the fundamental component for indirect generation of a reference [47]. In fact the neural network tracks all of the terms of the harmonic series but only the fundamental is then used. At each sample point, the predicted load current (generated from Fourier terms) is compared with the measured load current. The error is used to adapt the network weights. Simulation results in [47] show convergence of the estimated fundamental in 2-3 fundamental cycles. Following a step change in load, good performance is noted in the following cycle. The sample rate used was high $(12.5 \mathrm{kHz})$ and there remains the question over whether the neural network adaptation and recall can be completed at that rate in real time and whether the computational effort compares favourably with analytic techniques in sections 5.1 to 5.3.

The predict-ahead ability of neural networks is attractive for an APF correcting a load in transient. In [48] a neural network was trained to predict the fundamental component of load current for the next period given input data from the load. The example given was the DC current and firing angle of the thyristor bridge load. This may be feasible for an APF dedicated to a specific load but is not generally applicable. Another approach in [49] uses current data from a rectifier load to predict the fundamental active and reactive currents on a sample by sample basis. It was shown that the neural network is sufficiently small for real-time computation.

\section{Instantaneous Power Compensation}

One view of an active power filter is that it should cancel the fluctuating component of instantaneous power and perhaps also fundamental frequency reactive power. A common approach is to use the definitions of instantaneous active power as traditionally defined and the definition of instantaneous reactive power introduced by Akagi [50]. The definitions apply in either the $\alpha \beta 0$ - or $d q 0$-domains and for balanced sinusoidal three-phase systems would yield constant values. 
There are various representations of the equations such as complex power or a two-dimensional cross product. In the $\alpha \beta$-domain (i.e., without the zero-sequence term):

$$
s=p+j q=v_{\alpha \beta} \cdot i_{\alpha \beta}^{*}=\left(v_{\alpha}+j v_{\beta}\right)\left(i_{\alpha}-j i_{\beta}\right)=\left(v_{\alpha} i_{\alpha}+v_{\beta} i_{\beta}\right)+j\left(v_{\beta} i_{\alpha}-v_{\alpha} i_{\beta}\right)
$$

where * is used to denote a complex conjugate.

$$
\left[\begin{array}{l}
p \\
q
\end{array}\right]=\left[\begin{array}{l}
\left(v_{\alpha} i_{\alpha}+v_{\beta} i_{\beta}\right) \\
\left(v_{\beta} i_{\alpha}-v_{\alpha} i_{\beta}\right)
\end{array}\right]=\left[\begin{array}{cc}
v_{\alpha} & v_{\beta} \\
v_{\beta} & -v_{\alpha}
\end{array}\right]\left[\begin{array}{l}
i_{\alpha} \\
i_{\beta}
\end{array}\right]
$$

or in the $d q$-domain:

$$
s=p+j q=v_{d q} \cdot i_{d q}^{*}=\left(v_{d}+j v_{q}\right)\left(i_{d}-j i_{q}\right)=\left(v_{d} i_{d}+v_{q} i_{q}\right)+j\left(v_{q} i_{d}-v_{d} i_{q}\right)
$$

In a 4-wire system there is an additional term for the zero-sequence instantaneous power. In [51] this was allocated to active power but more recent work recognises the non-active element of the zero-sequence term [52].

It is common to show the instantaneous powers as composed of a steady average term and a variation (or oscillation) around this.

$$
\begin{aligned}
& p(t)=\bar{p}+\tilde{p}(t) \\
& q(t)=\bar{q}+\tilde{q}(t)
\end{aligned}
$$

Taking the case of an undistorted supply voltage but a harmonically distorted and unbalanced current (as used in Figure 4), the spectrum of the instantaneous power has the form shown in Figure 6. It is, therefore, relatively easy to use a filter to extract the fundamental frequency real and reactive powers. For a current composed of an in-phase fundamental and a single harmonic term the instantaneous power is:

$$
\begin{aligned}
& p=V_{1} I_{1}+V_{1} I_{n} \cos ((1-n) \omega t) \\
& \bar{p}=V_{1} I_{1} \quad \tilde{p}=V_{1} I_{n} \cos ((1-n) \omega t) \\
& q=-V_{1} I_{n} \sin ((1-n) \omega t) \\
& \bar{q}=0 \quad \tilde{q}=-V_{1} I_{n} \sin ((1-n) \omega t)
\end{aligned}
$$


Characteristic harmonic distortion (of order $\mathrm{n}=6 k+1$, i.e.. $-11^{\text {th }},-5^{\text {th }}, 7^{\text {th }}, 13^{\text {th }}$ etc., where negative orders represent negative sequence sets) yields power terms of order $6|k|$. Non-characteristic harmonic distortion (of harmonic order $\mathrm{n}=6 k$-1, i.e., $-13^{\text {th }},-7^{\text {th }}, 5^{\text {th }}, 11^{\text {th }}$ etc.) gives additional power terms at $6|k| \pm 2$.

The inverse transforms for conversion from the power domain to the phase domain is defined as:

$$
i_{\alpha \beta}^{*}=i_{\alpha}-j i_{\beta}=\frac{s}{v_{\alpha \beta}}=\frac{\left(p v_{\alpha}+q v_{\beta}\right)+j\left(q v_{\alpha}-p v_{\beta}\right)}{v_{\alpha}^{2}+v_{\beta}^{2}}
$$

or

$$
\left[\begin{array}{l}
i_{\alpha} \\
i_{\beta}
\end{array}\right]=\frac{1}{v_{\alpha}^{2}+v_{\beta}^{2}}\left[\begin{array}{cc}
v_{\alpha} & v_{\beta} \\
v_{\beta} & -v_{\alpha}
\end{array}\right]\left[\begin{array}{l}
p \\
q
\end{array}\right]
$$

Direct and indirect identifiers can be constructed as shown in Figure 7 following the principles established for the filtering techniques in section 5.1. Although not shown in the figure, the $p$ and $q$ terms are available separately and so one can choose whether the APF should or should not compensate the fundamental reactive power. In the direct case, the $q$-term is left unfiltered if reactive power compensation is required: in the indirect case the $q$-term is set to zero. Fundamental frequency negative sequence current appears as a second harmonic in the instantaneous power domain and is cancelled or not by the APF depending on the cut-off chosen for the identifier's filter.

The instantaneous power method applied in the $\alpha \beta 0$-domain is attractive because it has a low processing burden (no rotational transformation) and does not rely on explicit synchronisation.

The description so far has concentrated on a shunt filter correcting the current drawn from a voltage source. Instantaneous power decomposition can be used for the dual of this situation in which a series APF injects voltage to ensure constant instantaneous power when current flows into a voltage-distorting load [53]. The instantaneous power method has been applied to a series APF such that it passes only active power and seeks to block other terms [54]. This proposal also included additional injected voltage to correct voltage unbalance in the gird. 
An alternative formulation of instantaneous active and reactive powers using space vectors was reported in [55].

This and several other methods were compared in [56].

It should be emphasised that the preceding discussion assumed that the voltage was free of distortion. Several studies have noted that the instantaneous power method causes harmonic currents to flow when the voltage is distorted or unbalanced $[57,58,59]$. It is particularly important to note that unbalanced voltage can cause an APF controlled on instantaneous power to inject harmonic current. The relationship between the amplitude of the voltage distortion and the consequent current distortion was shown to be approximately proportional [60]. This study did not reveal the detailed relationship between the excitation and the response.

With a distorted grid voltage, a system drawing sinusoidal current would not have constant instantaneous power [53] and, as a corollary, an APF controlled on instantaneous power and subjected to distorted voltage can not achieve sinusoidal current. To maintain a constant instantaneous power by drawing a current from a harmonically distorted voltage requires a non-sinusoidal current. Similarly, a non-sinusoidal current is required to draw constant instantaneous power from a voltage containing a fundamental frequency negative sequence term.

It is worth noting that a balanced resistive load does not draw constant instantaneous power when supplied by a distorted 3-phase voltage set. Where supply voltage containing a balanced $n^{\text {th }}$ harmonic and a balanced fundamental voltage (19) is applied to a resistive load, an oscillatory power at the difference frequency is found to flow (24).

$$
\begin{aligned}
& v_{\alpha}=V_{1} \cos (\omega t)+V_{n} \cos (n \omega t) \\
& v_{\beta}=V_{1} \sin (\omega t)+V_{n} \sin (n \omega t) \\
& i_{\alpha}=\frac{V_{1}}{R} \cos (\omega t)+\frac{V_{n}}{R} \cos (n \omega t) \\
& i_{\beta}=\frac{V}{R} \sin (\omega t)+\frac{V_{n}}{R} \sin (n \omega t) \\
& \bar{p}=\frac{V_{1}{ }^{2}}{R}+\frac{V_{n}^{2}}{R} \\
& \tilde{p}=2 \frac{V_{1} V_{n}}{R} \cos ((1-n) \omega t)
\end{aligned}
$$


If an APF using a constant instantaneous power approach was applied to this situation then the APF would "correct" the current drawn by the resistor. The supply current corresponding to constant power and distorted voltage would be:

$$
\begin{aligned}
& i_{\alpha}=\frac{P v_{\alpha}}{v_{\alpha}{ }^{2}+v_{\beta}{ }^{2}}=\frac{P\left(V_{1} \cos (\omega t)+V_{n} \cos (n \omega t)\right)}{V_{1}{ }^{2}+2 V_{1} V_{n} \cos ((1-n) \omega t)+V_{n}^{2}} \\
& i_{\beta}=\frac{-P v_{\beta}}{v_{\alpha}{ }^{2}+v_{\beta}{ }^{2}}=\frac{P\left(V_{1} \sin (\omega t)+V_{n} \sin (n \omega t)\right)}{V_{1}{ }^{2}+2 V_{1} V_{n} \cos ((1-n) \omega t)+V_{n}^{2}}
\end{aligned}
$$

Both the numerators and denominators in these equations contain harmonic terms. Interestingly, the current harmonics created by these functions are not of the same order as the voltage excitation and a single voltage harmonic gives rise to an infinite series of current harmonics. In the case of negative sequence voltage unbalance (the presence of an $n=-1$ term), the inverse transform introduces unbalance in the currents and harmonic distortion, as was observed in [57].

In [58] it was suggested that the inverse transform should be changed so that the correction power is divided by a sinusoidal voltage set of the same amplitude as the fundamental voltage component. This prevents voltage distortion causing current distortion and means that the APF + Load presents a high impedance to voltage distortion. It is also noted as part of the discussion in [27] that the (positive sequence) fundamental component of the gird voltage should be used in the calculation of active power if the requirement is to obtain sinusoidal current after compensation. A different approach is taken in $[61,62]$ in which alternative definitions of $p$ and $q$ are used. The advantage is that the denominator term in the inverse transform is more nearly constant when the voltages are distorted or unbalanced. The implementation requires elements able to time-shift the instantaneous voltages by a quarter of the fundamental period.

If the inverse transform (17) were modified to use the mean square current, e.g. (27), then the denominator would not by time varying and would not introduce modulation of the current. This will have so modified the method that it is now equivalent to the definition of active power in (1) and is the basis of the impedance synthesis methods to be discussed in section 7.2.

$i_{\alpha}=\frac{\bar{p} v_{\alpha}}{\left\|v_{\alpha}\right\|+\left\|v_{\beta}\right\|}$ 
A method that bears some similarity to the instantaneous power method and to the $d q 0$ transformation is reported in $[63,64]$. No phase-locked loop is used; instead current signals are rotated by an angle derived from the instantaneous voltages $\left(\theta=\tan ^{-1}\left(v_{\alpha} / v_{\beta}\right)\right)$. This can also be expressed as:

$$
\left[\begin{array}{l}
i_{d^{\prime}} \\
i_{q^{\prime}}
\end{array}\right]=\frac{1}{\sqrt{v_{\alpha}{ }^{2}+v_{\beta}^{2}}}\left[\begin{array}{cc}
v_{\alpha} & v_{\beta} \\
-v_{\beta} & v_{\alpha}
\end{array}\right]\left[\begin{array}{l}
i_{\alpha} \\
i_{\beta}
\end{array}\right]
$$

These currents were described as instantaneous active and reactive current but not in the sense of [22]. In a similar manner to instantaneous active and reactive power, these currents can be decomposed into steady and oscillatory terms. Results in [63] show that under unbalanced and non-sinusoidal voltage conditions the proposed method offers a lower total harmonic distortion than the instantaneous power method but does not eliminate harmonics or provide a resistive characteristic. Under unbalanced voltage, harmonic distortion is created but to as lesser extent than in instantaneous power methods.

\section{Impedance Methods}

There have been two themes of development in which an APF is controlled so that alone, or in combination with another element, voltage and current are related by a chosen value of impedance.

\subsection{Impedance-Based Blocking}

A traditional filter arranges shunt and series elements with high and low impedance values to block or enable a signal path. An inverter can be made to act as an impedance (by, for instance, relating its voltage to its current by a simple transfer function) and can form one element of a filter. For an active power filter, this normally takes the form of a hybrid of active and passive elements although combinations of active units are also possible. There are many forms of hybrid filter and a discussion of their relative merits is outside the scope of this work. Here they will be discussed simply to illustrate the use of impedance-based blocking

In [65] and in [31] a series APF is used together with a shunt passive filter as part of a hybrid filter. The role of the series APF is to block harmonic current by presenting a high impedance. The method used in [65] is based on instantaneous power theory to separate the distortion terms in the current. The APF then injects voltage 
proportional to this current to synthesise moderately high impedance. It is noted that the filter used to separate the oscillatory terms in the instantaneous power will not be perfect and will influence the impedance presented by the overall APF. The method in [31] is to operate the series element as a controlled current source. A filter (in a synchronous frame) is used to identify the fundamental frequency current which is allowed to flow un impeded. Any other current flowing is treated as an error, which when multiplied by the controller gain, will produce an opposing voltage. The approach taken in [66] is to use the series APF to follow a sinusoidal template. Analysis of the response of the current control loop shows that it presents an impedance to higher harmonics terms and that proper design of this controller element means that it forms an effective filter in combination with passive filter elements. Methods in which the injected series voltage is made proportional to the current are described as supply current detection in [6].

\subsection{Impedance-Based Compensation}

A difficulty faced in designing an APF for general application is that the dynamics of the system into which the APF is introduced are unknown. This is a particular problem with an APF correcting a group of loads supplied via a section of distribution network. The network will contain both inductive and capacitive elements and therefore is resonant at one or more frequencies. The correction current, which is injected by an APF into the network in response to the detected current distortion, perturbs the voltage and can cause instability [67]. An alternative approach is to arrange a shunt element to draw a current proportional to the voltage. This is described as voltage detection in [6] and [67]. An interesting view of this problem follows from ideas of passivity [28]. The APF is configured so that it is dissipative (i.e., energy absorbing) at harmonic frequencies such that voltage harmonics detected in the network are damped and reduced in amplitude. The controller is passive in that it presents a resistive characteristic. The operation of the APF is stable regardless of line structure and parameters. The controller of [28] detects the load voltage and then sets a current reference in proportion. It works in the frequency domain using a filter bank to detect each harmonic term of interest. With a sufficiently low value of synthesised resistance at a particular frequency, the harmonics can be reduced to acceptably small values. An additional technique is introduced so that the harmonics can be eliminated if the line structure and parameters are known. Results are shown to demonstrate the instability due load dynamics in a standard APF and further results show zero steady-state error in an adaptive version of the passivity design. 
From a different stand point, an alternative technique based on a resistive characteristic is obtained [29]. The starting point is a recognition that correcting a load so that it draws sinusoidal current means that the load + APF presents infinite impedance to harmonic voltages and therefore provides no damping. A distribution system containing an inductive-capacitive resonance relies on load damping to attenuate the resonance. The approach proposed is to control the load + APF current to synthesise a resistance. This is achieved by defining the ratio between the instantaneous voltage and current. The value of that resistance is set such that the power requirement of the load is met. This objective is achieved by adjusting the resistance in response to error in the DC-bus voltage of the APF. Similarly, a single-phase APF compensating several loads has been described [68] in which the supply current is forced to follow a reference current of the same shape as the grid voltage. Again, the reference shape is scaled in response to error in the DC-bus voltage.

A single-phase impedance scheme has been reported in which the voltage of the shunt APF is made to have a resistive relationship to the overall supply current [69]. The APF is connected via a coupling inductor and forms one branch of the filter. Results in [69] show that the supply current is corrected to approximately the same harmonic distortion as the grid voltage.

\section{DC-Bus Energy Balance}

The DC-bus of the inverter in an APF is not a DC-link but an energy store. It is normal to use a voltage-source inverter and so the link contains a capacitor. The link voltage will remain constant provided there is no real power exchange between the APF and the AC-grid and provided that the power converter operates without losses. Neither condition is realised in practice. Power loss in the power converter (through conduction and switching losses) must be compensated by drawing a balancing power into the DC-bus from the AC-grid. Loss balancing is normally achieved through a simple DC-bus voltage regulator such as Figure 8. Voltage error is acted on by a controller, the output of which is taken as a demand for fundamental frequency active current. This term is added to the compensation reference. The control can take place in any convenient domain.

The second reason that the DC-bus may deviate from its reference is that the compensation reference falsely includes a real power component. Typically this will be an in-phase fundamental frequency component that arises from phase or magnitude error in the distortion identifier or from time-delay in responding to a transient condition. There will also be some error between the identified correction current and the current the inverter 
actually achieves and this may lead to power exchange. Another possibility is that the grid voltage is distorted and real power is being exchanged through harmonic terms. The DC-bus regulator must be designed to make up for these deficiencies in the distortion identification.

It is important to choose the bandwidth of this controller carefully. If the bandwidth is set too high (comparable with the fundamental frequency) then the balancing current injected by this controller will contain components at harmonic frequencies and will add distortion to the system. If the bandwidth is set too low then the DC-link voltage may deviate too far from its nominal value. An upswing of voltage may exceed the ratings of the inverter switches and a downswing might leave insufficient voltage for the APF to inject the correct compensation. A PI compensator operating on the voltage error is often used and can be easily arranged to have a low cross-over frequency so as to avoid interaction with the compensation system. A predictive (dead-beat) method of DC-bus voltage control was given in [70].

In a single-phase APF there is an additional consideration in regulation of the DC-bus: the fundamental frequency reactive power of the load, if compensated, is exchanged with the DC-bus as a double frequency term. The regulator should not respond to voltage ripple at this double frequency term. Similarly, if a threephase APF is arranged to correct negative sequence fundamental current, there will be a double frequency voltage ripple on the DC-bus which should not be responded to by the DC-bus regulator.

The DC-bus regulator can make up for deficiencies in effective separation of active and non-active current in the distortion identifier. Taken to its extreme, the distortion identifier can be omitted altogether. In this case, the main APF controller is set to eliminate all components of the load current. The APF will then seek to supply the real power of the load and the DC-bus regulator will respond by drawing a balancing real power from the AC grid. The advantage of this method is that the processing burden of the distortion identifier is avoided. It is still necessary to control the injected current with a feedback loop. The disadvantage is that the APF cancels everything except the long-term average real power. Thus the APF must be rated sufficiently for the total burden of harmonics, unbalance, reactive power, flicker and transient real power. A variation of this scheme has been described $[58,59]$ in which the DC-energy balance term is used to set the amplitude of a reference set of supply waveforms. The inverter is then placed in closed-loop control to force these currents to flow in the supply. A 
similar approach is used in [71] but with a fuzzy element providing the control of the DC-bus voltage level. The use of DC-bus voltage control in impedance compensation was noted in section $7.2[29,68]$

DC energy balance methods of harmonic identification are popular in single-phase systems where some of the control schemes that exploit the properties of three-phase systems are not available. In [72] a PI compensator acting on the DC bus voltage error is used to identify the amplitude of the fundamental active current component required by the load. When multiplied by a unit sinusoid, this gives the reference value for the supply current. The supply current is then controlled in closed loop to follow this reference by applying the current error to a PWM controlled bridge. The relationship between the DC-bus capacitor size, the allowed voltage deviation and the controller design is also described in [72].

There is a special consideration for 4-wire systems. If there is zero-sequence voltage and zero-sequence current of the same frequency (commonly fundamental frequency but this can also apply to harmonics) then there is real power carried in the zero sequence set. This will show as zero sequence power under the instantaneous power transform. If the APF is set to cancel this term in the supply then the APF will need to source this real power for delivery to the load [53]. This requires an additional positive sequence power term to be drawn from the supply to maintain the DC-bus balance.

\section{Reference Following}

In the discussion so far it has been assumed that once identified, the current to be injected can be accurately achieved. Control of current injected by the APF such that if follows the identified reference is challenging because of the high rates-of-change and the wide bandwidth of that reference [73]. The errors in reference following introduced by the $d i / d t$ limit of a voltage source inverter were investigated in [74]. The limits arise from the interface inductance between the inverter and the line and the voltage that can be imposed by the inverter. The imposed voltage is the difference between the available DC-bus voltage and the instantaneous voltage of the AC system. Increasing the DC-bus voltage to improve the reference following has a direct impact on the ratings of the inverter. There are also constraints on the choice of interface inductor. It plays a dual role. First, it defines the current given the imposed voltage from the inverter and second it is a filter (or part of a filter) that attenuates the switching frequency voltage components created by the inverter such that acceptably 
small switching frequency current is injected into the AC system. Better reference following would compromise the attenuation of the switching frequency components. Also discussed in [74] is the effect of processing delay on the formation of the reference waveform. The delay is composed of signal acquisition, computation and signal output delays. Even small delays in this signal path can create significant errors because the delay represents a significant phase displacement of high-order harmonic terms. It was shown that delays of $28 \mu \mathrm{s}$ (in a $50 \mathrm{~Hz}, 128$ sample-per-cycle system) cause significant mis-cancellation of distortion terms. A different view of delays affecting APF accuracy is given in [16]. Here it is argued that delay in the reference following controller can be compensated by applying compensating time shifts to the individual harmonic terms in a frequency domain identification of the reference. Similarly, it was argued in [75] that each harmonic term could be adjusted for identification delay in order to provide better tracking of the correct compensation current.

Three approaches are available to force the output current of an inverter to follow a reference: current regulated PWM; hysteresis regulation and dead-beat/ predictive control [76]. The discussion here will focus on the control of voltage source inverters although there has been discussion of the use of current source inverters [77].

\subsection{Current Regulated PWM}

Current regulated PWM is well established in many application areas. The current error is acted on by, typically, a PI element and the result used in a standard pulse-width modulator or space-voltage vector modulation to set the voltage synthesised by the inverter. The controller can act in any convenient domain. The design of a current-regulated PWM system is described in [78] for closed loop control of grid currents in a single-phase APF. Control in the $d q 0$ domain including feed-forward of the AC voltage is combined with SVM in [47].

\subsection{Hysteresis}

In general, hysteresis regulation has the advantages that it is unconditionally stable, does not require detailed plant data and can achieve high rates-of-change in the controlled variable. These advantages are attractive in APF application where the reference can have sharp transitions and the network into which the APF injects is not perfectly known. A comparison was undertaken in [79] that concluded that the fast response and absence of following error of hysteresis control is advantageous in APF applications. The acknowledged disadvantage of hysteresis control is the broadband switching noise injected by the non-constant switching frequency. Several approaches have been explored to maintain the switching frequency constant (or nearly so) and to synchronise 
the switching of all phases. The application of these methods to an APF and comparison with other control methods is given in [73]. This paper also proposes a combination of feed-forward and feed-back (PLL) methods for setting the hysteresis bands to achieve a constant switching frequency.

\subsection{Dead-beat and Predictive Control}

Dead-beat or predictive control applied to current regulation aims to choose a voltage command for an inverter that will cause the current to reach a target value by the end of the next sample period and is therefore attractive in following rapidly changing references in an APF [19]. It requires knowledge of the system so that the rate-ofchange of current can be accurately modelled and the voltage chosen. The interface inductance of the inverter is a key parameter and the opposing voltage (the AC system voltage in this case) must be measured or estimated. A development of a predictive current controller is described in [76]. This is a general method, not specific to an APF, and is developed in two variants for either measured or estimated EMF. Dead-beat control was considered in [80] for the case where there is parameter uncertainty with particular reference to the value of the coupling inductor. The underlying control is a method that forms a resistive total load. Stability limits of current controller under parameter mismatch were described and the influence of the APF control properties examined. In [81] an adaptive FIR filter is used to predict ahead the current reference so that the dead-beat controller uses the predicted current at the end of the sample period as the target current.

\section{Discussion}

Mitigating harmonics is a key function of an active power filter but there are three competing definitions of the basis for form of current that should remain in the distribution network after mitigation and hence the approach taken to mitigation. The options are: (i) the current should contain only fundamental frequency components, (ii) the current should be such that the instantaneous active power is constant and instantaneous reactive power is zero and (iii) the current should be entirely active current (as defined by Fryze). If it is assumed that the distribution network voltage is free of harmonic distortion and unbalance then these three objects are equivalent and in much of the published work the distinction between the objectives is not made explicit. In choosing one objective over another as the basis for APF control, ease of implementation and the instrumentation requirement can be allowed to dominate if the supply is distortion free. The waveform compensation (fundamental-only) method is the obvious approach given the use of harmonic distortion limits in the relevant standards. The major issue is the implementation of the filtering to identify fundamental from harmonics. Time domain filters have 
advantages over frequency domain filters for continuity during transients but frequency domain filters can achieve better selectivity in steady state. In three-phase systems, the synchronous reference frame ( $d q$ transform) can ease the filter implementation at the expense of some processing overhead. The instantaneous power method is advantageous in three-phase systems because of the ease of filter design without the need of a $d q$ transform. The active current approach requires identification of the real (average) power and so is a relatively simple filtering task. This approach is implicit in methods that use energy balance of the DC bus voltage as the basis of identifying the desired current.

Product standards and testing methods for harmonic distortion often assume a "clean" supply and all three approaches are successful. However, important issues arise when APFs are operated on weak sections of distribution network where the voltage is distorted. From a network operator's point of view, the performance of the APF can affect the propagation of harmonic voltages through the network. From the consumer's point of view, the distorted voltage may increase the apparent power requirement of the APF. It would be desirable to encourage consumers to adopt a "model citizen" approach and configure APFs to contribute to a better electrical environment across the network. An APF operated to draw fundamental current only presents an open circuit to harmonic voltage excitation. This does not contribute extra distortion but neither does it provide damping of the existing distortion in the way that traditional resistive loads would. Instantaneous active power methods respond to harmonic voltages by introducing additional harmonic currents at other frequencies and could produce unfortunate interactions with resonances in the network. Most notably, negative sequence harmonic voltage causes the APF to inject harmonic current. The active current APF presents a resistive characteristic at all frequencies and therefore converts non-linear loads into linear loads. Thus, the network is provided with damping to reduce the propagation of harmonic voltages. If this approach is to be exploited there will need to be some coordination of the design of APFs with the existing approaches to the damping of network resonances employed by network operators. As yet this form of load current response is not addressed by standards.

Harmonic mitigation is not the only objective or response of interest in an APF. Unbalanced load currents can be rebalanced provide the exchange of instantaneous power between the phases is within the rating of the APF. All approaches to compensation can rebalance current when implemented in three-phase form. Waveform compensation and active current methods can be implemented on a phase-by-phase basis to specifically avoid rebalancing. Unbalance from the network voltage must also be examined and it was already noted that 
instantaneous power methods have an unacceptable response. Modifications have been proposed to lessen or remove the harmonic generation through redefining the inverse transform. Taken to their conclusion, these modifications result in a method close to one of the other known approaches. Fundamental-only methods result in balanced current despite unbalanced voltage whereas active current methods present a balanced impedance to the unbalanced voltage and hence draw least current from the lowest phase voltage. The constant instantaneous power method would draw greatest current from the lowest phase voltage.

\section{Conclusions}

The many competing control techniques for active power filters have been compared and discussed under three broad approaches to distortion compensation. There are implementation advantages of some methods over others but they can produce broadly similar performance if the supply voltage is undistorted. When applied in practice, distortion and unbalance of the network voltage will make the performance of the instantaneous power method (in unmodified form) unacceptable. The fundamental-only compensation method will meet the necessary standards but does not take the next step of contributing to improving the network voltage. Methods based on achieving active-only current, which is equivalent to presenting a resistive characteristic at all frequencies, could contributed to voltage quality in the network if network planning could incorporate this feature. The choice of control objective and the filtering approach taken to separate desired from undesired components of current needs careful examination in all cases to ensure that the terms explicitly or implicitly compensated, which might include harmonics, fundamental reactive power, fundamental negative sequence, zero sequence and flicker originating from either the non-linear load or the distorted network voltage, can be accommodated within the apparent power rating of the active filter. The choice of domain and reference frame in which to implement the filter affects the ease with which certain components can be accurately identified and separated in cases where pass and stop bands are in close proximity, however, the processing overhead and dependence on three-phase configuration limits the choice in some cases. 


\section{References}

1 H. Sasaki and T. Machida, "A new method to eliminate AC harmonic current by magnetic flux compensation - considerations on basic design”, IEEE Trans. on Power Sys. And Apparatus, Vol. 90, No. 5, pp. 2009-2019, 1971

2 L. Gyugyi and E. Strycula, “Active AC power filters” IEEE Ind. Appl. Soc. Ann. Mtg., pp. 529-535, 1976

3 A. Ametani, "Harmonic reduction in thyristor converters by harmonic current injection", IEEE Trans. on Power Sys. And Apparatus, Vol. 95, No. 2, pp. 441-449, 1976

4 N. Mohan, H.A. Peterson, W.F. Long, G.R. Dreifuerst and J.J. Vithaythil, “Active filters for AC harmonic suppression”, IEEE Winter Power Meeting, 1977

5 W.M. Grady, A.H. Noyola, and M.J. Samotyj, "Survey of active power line conditioning methodologies", IEEE Trans. on Power Delivery, Vol. 5, No. 3, pp. 1536-1542, 1990

6 H. Akagi, "New trends in active filters for power conditioning”, IEEE Trans. on Ind. Appl., Vol. 32, No. 6, pp. 1312-1322, 1996

$7 \quad$ F.Z. Peng, “Applications issues of active power filters", IEEE Industry Applications Magazine, Vol. 4, No. 5, pp. 21-30, 1998

8 T.C. Green and J.H. Marks, "Issues in the Rating of Active Power Filters", IEE Proc. Electric Power Applications, Vol. 150, No. 5, pp. 607-614, 2003

9 F.Z. Peng, "Harmonic sources and filtering approaches", IEEE Industry Applications Magazine, Vol. 7, No. 4, pp. 18-25, 2001.

10 S.T. Senini and P.J. Wolfs, "Systematic identification and review of hybrid active filter topologies", IEEE Power Elec. Specialist Conf., pp. 394-399, 2002

11 B. Singh, K. Al-Haddad and A. Chandra, "A review of active power filters for power quality improvement", IEEE Trans on IE, Vol. 46,No. 5, pp 960-971, 1999

12 M. El-Habrouk, M.K. Darwish and P. Mehta, “Active power filters: a review”, IEE Proc. EPA, Vol. 147, No. 5, pp. 403-413, 2000

13 L.A. Pittorino, A. Horn and J.H.R. Enslin, "Power theory evaluation for the control of an active power filter", IEEE, pp. 676-681, 1996.

14 G.D. Marques, "A comparison of active power filter control methods in unbalanced and non-sinusoidal conditions", IEEE, pp. 444449,1998

15 S. Rechka, E. Ngandui, J.Xu and P. Sicard, "A comparative study of harmonic detection algorithms for active filter and hybrid active filers", IEEE Power Elec. Specialist Conf., pp. 357-363, 2002

16 S. Mariethoz and A.C. Rufer, "Open loop and closed loop spectral frequency active filtering", IEEE Trans on Power Electronics, Vol.17, No. 4, pp. 564-573, 2002

17 A.D.le Roux, J.A. du Toit and J.H.R. Enslin, "Integrated rectifier and power quality compensator with reduced current measurement", IEEE Trans on IE, Vol. 46, No. 3, pp. 504-511, 1999

18 L. Malesani, L. Rossetto and P. Tenti, "Active filters for reactive power and harmonic compensation", IEEE Power Electronics Specialist Conf. (PESC'86), pp.321-330, 1986

19 J.H. Allmeling, "A control structure for fast harmonic compensation in active filters", IEEE Power Electronics Specialist Conf. (PESC`02), pp. 376-381, 2002 
20 A. Nabae and M. Yamaguchi, "Suppression of flicker in an arc-furnace supply system by an active capacitance-a novel voltage stabilizer in power systems", IEEE Trans on IA, Vol., 31, No. 1, pp. 107-111, 1995

21 H. Rudnick, J. Dixon and L. Morán, “Delivering clean and pure power”, IEEE Power \& Energy Magazine, Sept./Oct. pp. 32-40, 2003

22 S. Fryze, “Active, reactive and apparent power in non-sinusoidal systems”, Przeglad Elekrot, No. 7, pp. 193-203, 1931 (in Polish)

23 J.H.R. Enslin and J.D. van Wyk, "Measurement and compensation of fictitious power under non-sinusoidal voltage and current conditions", IEEE Trans on Instrumentation and Measurement, Vol. 37, No. 3, pp. 403-408, 1988

24 J.H.R. Enslin and J.D. Van Wyk, "A new control philosophy for power electronic converters as fictitious power compensators”, IEE Trans on PE, Vol. 5, No. 1, pp. 88-97, 1990

25 L.S. Czarnecki, “Distortion power in systems with non-sinusoidal voltage”, IEE Proc. Pt. B, Vol. 138, No. 3, pp. 276280, 1992

26 L.S. Czarnecki, "Comments on "Measurement and compensation of fictitious power under non-sinusoidal voltage and current conditions", IEEE Trans on Instrumentation and Measurement”, IEEE Trans on PE, Vol. 5, No. 4, pp. 503-504, 1990

27 F.Z. Peng, L.M. Tolbert and Z. Qian, "Definitions and compensation of non-active current in power systems", IEEE Power Electronics Specialist Conference (PESC '02), Cairns, Australia, pp. 1779-1784, June 2002.

28 P. Mattavelli and A.M. Stankovic, "Energy-based compensation strategy for active filters", CDC, Pheonix, December, pp. $4656-4661$ 1999

29 T.E. Núñez-Zúñiga and J.A. Pomilio, "Shunt Active Power Filter Synthesizing Resistive Loads", IEEE Trans on Power Electronics, Vol. 17, No. 2, pp. 273-278, 2002

30 S. Bhattacharya, D.M.Divan and B. Banerjee, "Synchronous frame harmonic isolator using active series filter", European Conf on Power Electronics and Applications EPE, pp. 30-35, 1991.

31 S. Bhattacharya and D.M.Divan, "Synchronous frame based controller implementation for series hybrid active filer system", IEEE, pp. $2531-2540,1995$

32 M.J. Newman, D.N.Zmood and D.G. Homes, "Stationary frame harmonic reference generation for active filters systems", IEEE Applied Power Electronics Conference (APEC 2002), pp. 1054-1060, 2002

33 H. Akagi et al., "Control strategy for active power filters using multiple voltage source PWM converters", IEEE Trans. on IA, Vol. 22, No. 3, pp. $460-, 1986$

34 H.-L. Jou, .-C. Wu and H.-Y. Chu, "New single-phase active power filter", IEE Proc EPA, Vol. 142, No. 3, pp. 129-134, 1995

35 J.S. Tepper, J.W. Dixon, G. Venegas and L. Morán, “A simple frequency independent method for calculating the reactive and harmonic current in a non-linear load”, IEEE Trans on IE, Vol. 43, No. 6, pp. 647-653, 1996

36 L.A. Pittorino, J.A. du Toit and J.H.R. Enslin, "Evaluation of converter topologies and controllers for power quality compensators under balanced conditions", IEEE, pp. 1127-1133, 1997

37 S. Lou and Z. Hou, “An adaptive detecting method for harmonic and reactive currents", IEEE Trans IE, Vol. 42, No. 1, pp. 85-89, 1995

38 C.D. Schauder and S.A. Moran, "Multiple reference frame controller for active filters and power line conditioners", US Patent 5309353,1994

39 P. Mattavelli, P. Tenti, "High performance active filters using selective harmonic control”, IEEE Power Engineering Society Summer Meeting, pp. 977-982, 2000 
40 S. Osowski, "Neural network for estimation of harmonic components in a power system", IEE Proc. GTD, Vol. 139, No. 2, pp. 129135,1992

41 A.Kumamoto, T. Hikihara, Y. Hirane, K. Oku, S. Tada, K. Mizuki and Y. Ogihara, "Suppression of harmonic voltage distortion by neural network controller”, IEEE Ind. Appl. Annual Mtg., pp. 754-761, 1992

42 S.D. Round and N. Mohan, "Comparison of Frequency and Time Domain Neural Network Controllers for an Active Power Filter", IEEE IECON '93, vol.2, pp. 1099-1104, 1993.

43 M. Kandil, S. Abdelkader, A. Elmitwally and M. El-Kateb, "A novel three-phase active filter based on neural networks and sliding mode control”, IEEE Industrial Elec. Conf (IECON '99), pp. 867-872, 1999

44 A. Elmitwally, S. Abdelkader and M. El-Kateb, "Neural network controlled three-phase four-wire shunt active power filter", IEE Proc GTD, Vol. 147 2, pp. $87-92,2000$

45 N. Pecharanin, H. Mitsui and M. Sone, "Harmonic Detection Using Neural Network", IEEE Intl. Conf. On Neural Networks, pp.923$926,1995$.

J.R. Varquez and P. Salmeron, "Active power filter control using neural network technologies”, IEE Proc. EPA., Vol 150, No. 2, pp. $139-145,2003$

47 M. Rukonuzzaman and M. Nakoka, "An advanced active power filter with adaptive neural network based harmonic detection scheme" IEEE PESC 01, Vancouver, pp. 1602-1607, 2001

48 Z. Lu and T.C. Green, 'Neural network based predictive control strategy of an active power filter for electric drives', PEVD 98, IEE Conf. Publ. No. 456, London, U.K., pp. 287-291, 1998.

49 J H Marks and T C Green, "Predictive transient following control of shunt and series active power filters", IEEE Trans on Power Elec., Vol. 17, No. 4, pp. 574-584 2002

50 H. Akagi, Y. Kanazawa and A. Nabae, "Instantaneous reactive power compensators comprising switching devices without energy storage", IEEE Trans. on IA, Vol. 20, No. 3, pp. 625-703, 1984

51 H. Akagi and A. Nabae, "The p-q theory in three-phase systems under non-sinusoidal conditions", European Transaction on Electric Power, Vol. 3, pp. 27-31, 1993

52 F.Z. Peng and J.S. Lai, "Generalised instantaneous reactive power theory for three-phase power systems", IEE Trans on Instrumentation and Measurement, Vol. 45, No. 1, pp. 293-297, 1996

53 M. Aredes and E.H. Watanabe, "New control algorithms for series and shunt three-phase, four-wire active power filters", IEEE Trans on PD, Vol. 10, No. 3, pp. 1649-1656, 1995

54 L. Morán, I. Pastorini, J. Dixon and R. Wallace, "Series active power filter compensates current harmonics and voltage unbalance simultaneously”, IEE Proc GTD, Vol. 147, No. 1, pp. 31-36, 2000

55 A. Nabae and T. Tanaka, "A new definition of instantaneous active-reactive current and power based on instantaneous space vectors on polar coordinates in three-phase circuits", IEEE Trans on Power Delivery, Vol. 11, No. 3, pp. 1238 -1243, 1996

L.M. Tolbert and T.G. Hablter, "Comparison of time-based non-active power definitions for active filtering”, CIEP 2000, Acapulco, Mexico, 15th-19th October, pp. 73-79, 2000

57 W. le Roux and J.D. van Wyk, "Evaluation of residual network distortion during compensation according to the instantaneous power theory", Euro. Trans on Elect Power, Vol. 8, No. 5, pp. 337-344, 1998

58 H.L. Jou, "Performance comparison of three-phase active power filter algorithms", IEE Proc GTD, Vol. 142, No. 6, pp. 646-652, 1995 
59 S. Huang and J. Wu, "A control algorithm for three-phase three-wired active power filters under non-ideal mains voltage", IEEE Trans on PE, Vol. 14, No. 4, pp. 753-760, 1999

60 V. Cárdenas, L. Morán, A. Bahamondes and J. Dixon, "Comparative study of real time reference generation techniques for four-wire shunt active power filters", IEEE , pp.791-796, 2003

61 Y. Komatsu and T. Kawabata, "A control method of active power filter in unsymmetrical and distorted voltage system" Power Conv Conf, Nagaoka, Japan, August, pp.161-168 1997

62 Y. Komatsu and T. Kawabata, "A control method of active power filter in unsymmetrical and distorted voltage system", Int J electronics, Vol. 86, No. 10, pp. 1249-1260, 1999

63 V.Soares, P. Verdelho, G.D. Marques, “A control method for active power filters under non-sinusoidal conditions", IEE Conference on Power Electronics and Variable Speed Drives (PEVD '96), Conf. Pub. No. 429, London, pp. , 1996

64 V.Soares, P. Verdelho, G.D. Marques, “An instantaneous active and reactive current component method for active filters”, IEEE Trans on PE, Vol. 15, No. 4, pp. 660-669, 2000

65 F.Z. Peng, H. Akagi and A. Nabae, "Compensation characteristics of the combined system of shunt passive and series active filters", IEEE Trans on IA, Vol. 29, No. 1, pp. $144-152,1993$

66 J. Dixon, G. Venegas and L. Morán, “A series active power filter based on a sinusoidal current controlled voltage source”, IEEE Conf, pp. 639-644, 1995.

67 H. Akagi, "Control strategy and site selection of a shunt active filter for damping harmonic propagation in power distribution systems", IEEE Trans on Power Delivery, Vol. 12, No. 1, pp. 354-362, 1997

68 F. Pottker and I. Barbi, "Single phase active power filters for distributed power factor correction", IEEE Power Electronics Specialist Conf (PESC'00), Galway, Ireland, pp. 500-505, 2000

69 E. Dallago and M. Passoni, "Single-phase active power filter with only line current sensing”, IEE Electronic Letters, Vol. 36, No. 2, pp $105-106,2000$

70 C.Y. Hsu and H.Y Wu, "A new single-phase active power filter with reduced energy storage capacity", IEE Proc. EPA, Vol. 143, No. 1, pp. $25-30,1996$

71 J. Dixon, J. Contardo and L. Morán, "DC link fuzzy control for an active power filter sensing the line current only", IEEE pp. $1109-1114,1997$

72 J.C. Wu and H.L. Jou, “A simplified control method for single-phase active power filter” IEE Proc EPA, Vol. 143, No. 3, pp. 219-224, 1996

73 L. Malesani, P. Mattavelli and P. Tomasin, "High performance hysteresis modulation technique for active filters", IEEE Applied Power Electronics Conf. (APEC `96), pp. 939-946, 1996

74 W. le Roux and J.D. van Wyk, "Modeling of distortion compensation ineffectivity in filters for non-active power", IEEE Trans IE, Vol., 48, No. 1, pp. 91-100, 2001.

75 S. Jeong and M. Woo, "DSP-based active power filter with predictive current control”, IEEE Trans on IE, Vol. 44, No. ?, pp. 329-336, 1997

76 D.G. Holmes and D.A. Martin, "Implementation of a direct digital predictive current controller or single and three phase voltage source inverters”, IEEE Ind. Appl. Ann. Mtg., San Diego, USA, pp. 906-912, 1996 
77 E.P. Venter, J.D. van Wyk and L. Malesani, "A comparative evaluation of control strategies for current fed converters as filters for non-active power in networks", IEEE Ind. Appl. Annual Mtg, pp. 829-836, 4-9 Oct. 1992

78 F. Pottker and I. Barbi, "Power factor correction of non-linear loads employing single phase active power filter: control strategy, designing methodology and experimentation’, IEEE Power Electronics Specialist Conf (PESC'97), St. Luis, USA, pp.412-417, 1997

79 J.W. Dixon, S. Tepper and L.Morán, "Practical evaluation of different modulation techniques for current controlled voltage source inverters", IEE Proc EPA, Vol. 143, No. 4, pp. 301-306, 1996

80 L. Malesani, P. Mattavelli and S. Buso, "Robust dead-beat current control for PWM rectifiers and active filters", IEEE Conf, pp. 13771383,1998

81 K. Nishida, Y. Konishi and M. Nakaoka, "Current control implementation with dead-beat algorithm for three-phase current-source active power filter", IEE Proc EPA, Vol. 149, No. 4, pp. 275-282 


\section{Figures}

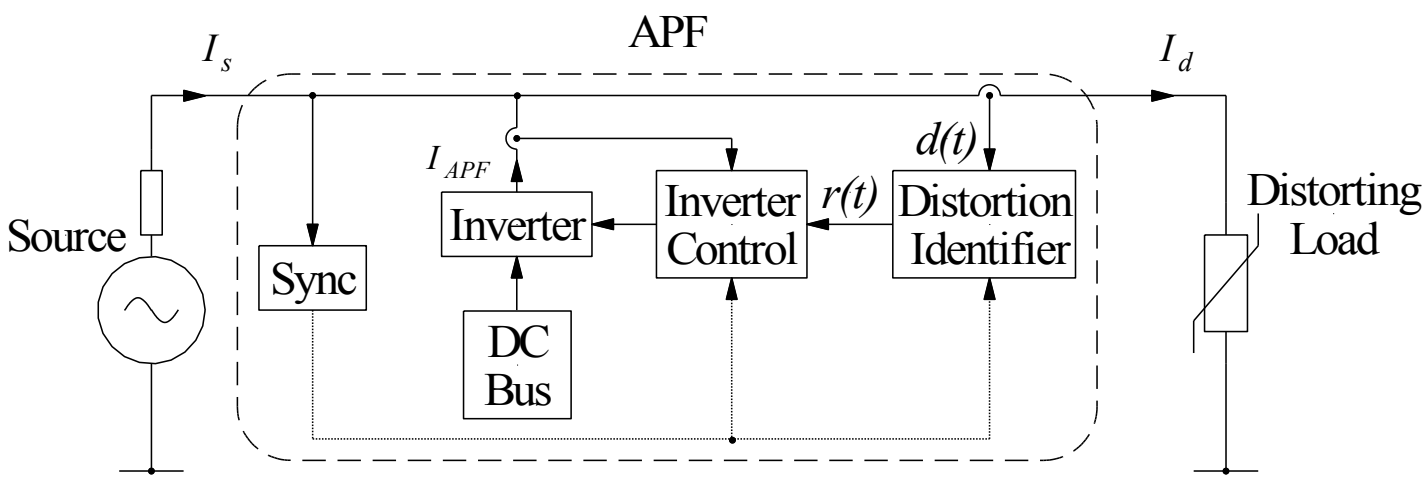

Figure 1 Schematic diagram of an example shunt APF

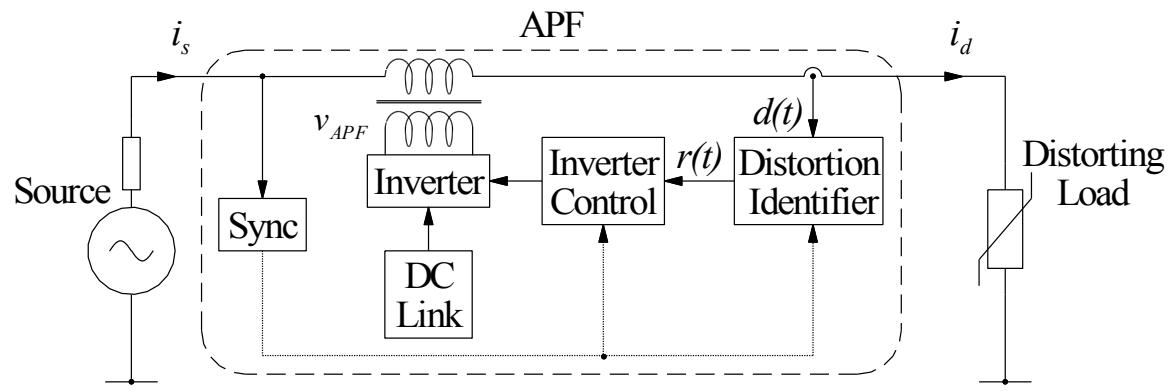

Figure 2 Schematic of an example series APF
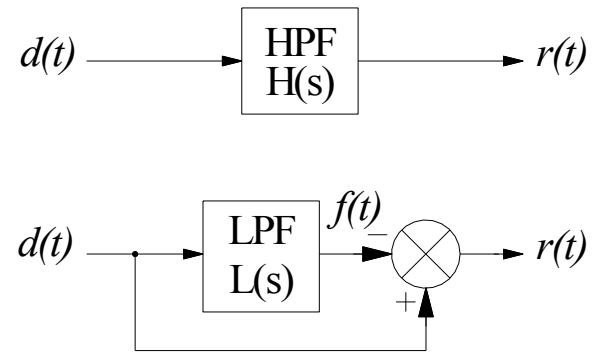

Figure 3 Direct and indirect distortion identifiers in the time domain. The signal $f(t)$ is the identified fundamental frequency signal that is allowed to remain un-cancelled. 


\begin{tabular}{|cc|}
\hline Characteristic & Non-Characteristic \\
Components & Components \\
Negative & Positive \\
Sequence & Sequence \\
Positive & Segative \\
Sequence & Nequence \\
\hline
\end{tabular}
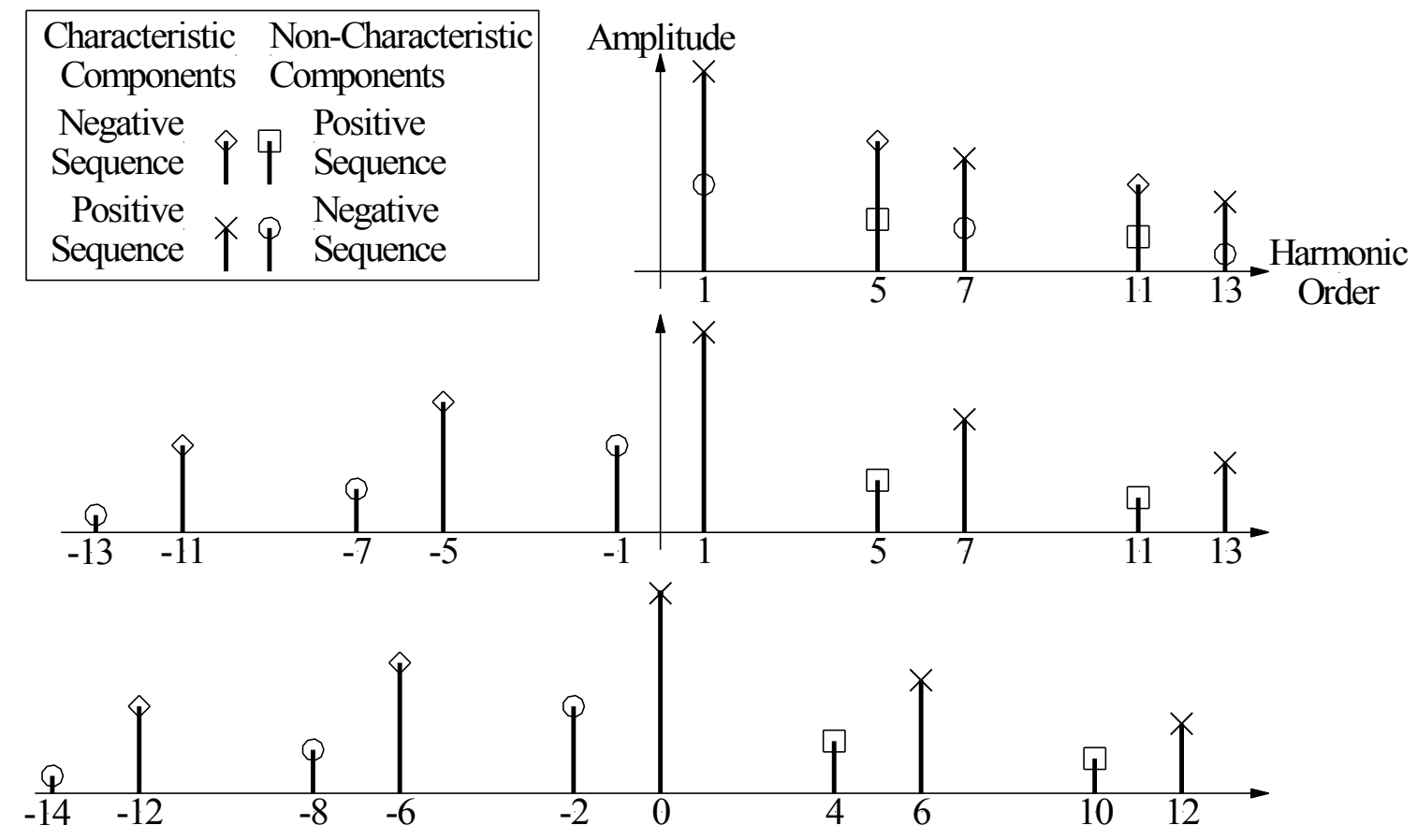

Figure 4 Spectrum of a distorted and unbalanced 3-wire, 3-phase signal in (a) phase,

(b) $\alpha \beta 0$ and (c) $d q 0$ domains.

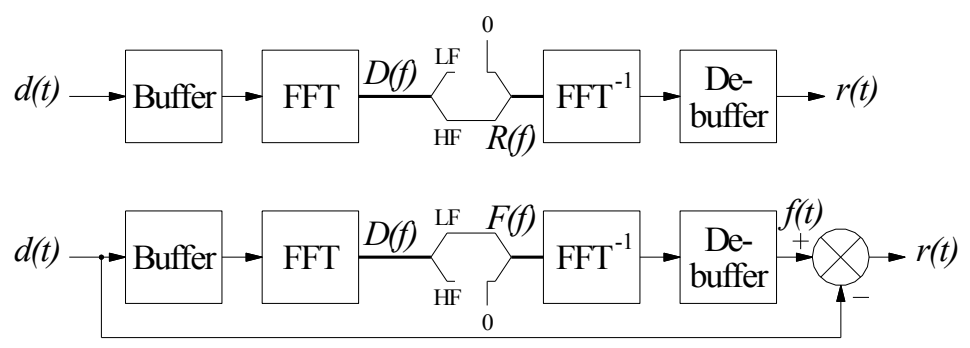

Figure 5 Direct and indirect filtering in the frequency domain. $D(f), R(f)$ and $F(f)$ are the frequency domain form of the signals $d(t), r(t)$ and $f(t)$ in figures 1 and 3 


\begin{tabular}{|cll|}
\hline Characteristic & Non-Characteristic \\
Component & Component \\
Negative & Positive \\
Sequence & Sequence \\
Positive & Negative \\
Sequence & Sequence \\
\hline
\end{tabular}
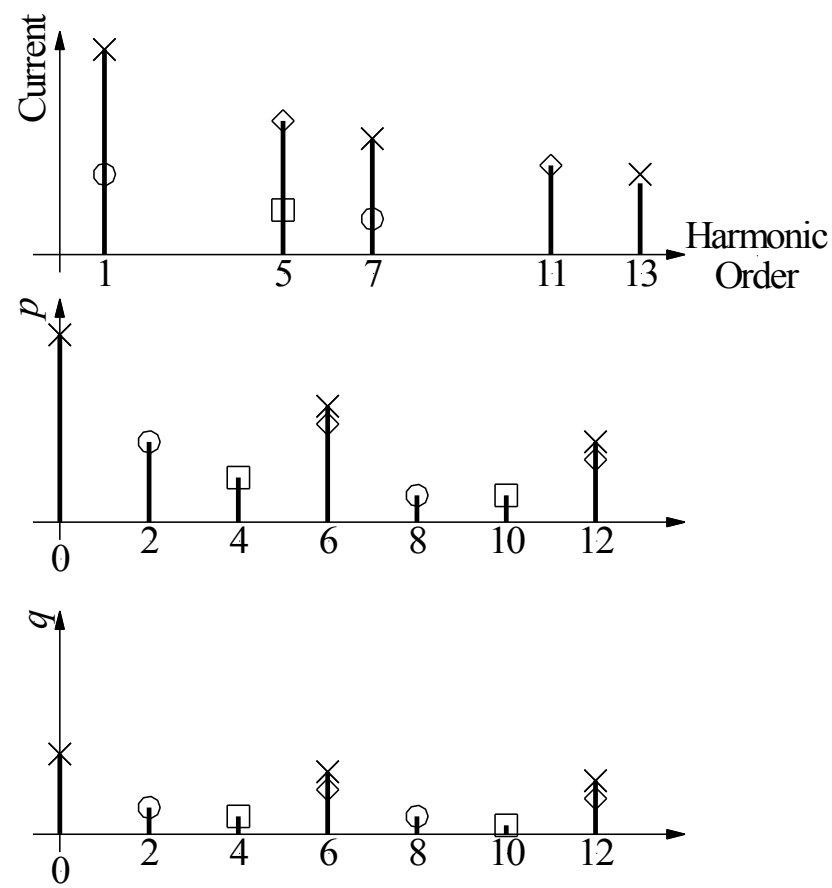

Figure 6 Phase-current and instantaneous power in the frequency domain.

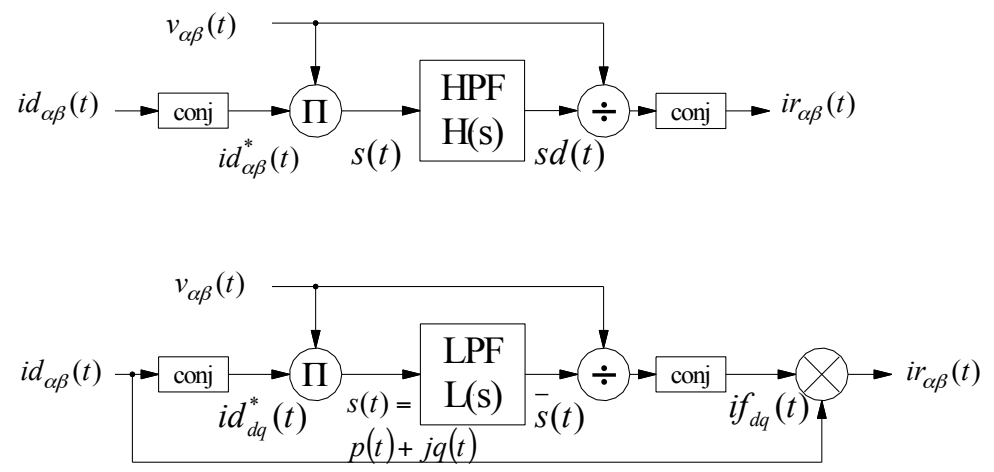

Figure 7 Direct and indirect distortion identifiers in the instantaneous power domain. 


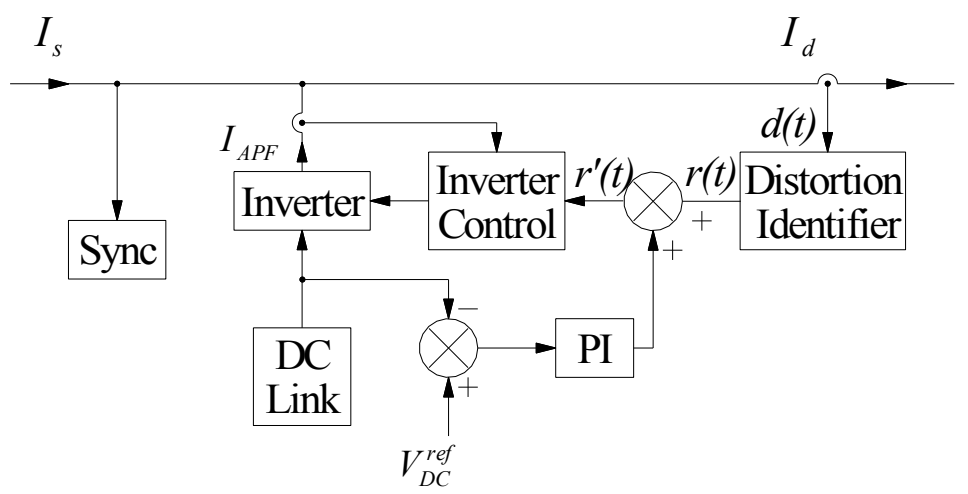

Figure 8 An example DC-bus voltage regulator 